\title{
Efficient Data Dissemination Techniques in VANETs: A Review
}

\author{
Bhawna Dhawan \\ M.Tech Research Scholar \\ Deptt. of CSE \\ PTU Regional Centre \\ ACET, Amritsar
}

\author{
Tanu Preet Singh, Ph.D. \\ HOD ECE \\ Deptt. of ECE \\ PTU Regional Centre \\ ACET, Amritsar
}

\begin{abstract}
Vehicular ad hoc network (VANETs) being a sub class of MANET is a challenging field of wireless communication. It is a sensible way of using infrastructure fewer networks augmented with Intelligent Transportation System (ITS). It is an emerging trend now days for vehicles to be equipped with an embedded on-board computing unit with communication capabilities to enhance the overall driving experience. Such a system enables vehicle to vehicle and vehicle-toinfrastructure communication and provides vehicles with upto-date route and traffic information. Due to its highly dynamic topology as well as occurring often disconnection among vehicles, various categories of protocols have designed for its efficient and reliable working. While driving, a large amount of data and information are accessible to everyone. Many attractive applications over vehicular ad hoc network (VANETs) need data to be transmitted to the remote destinations through multiple paths, but some unique characteristics of VANETs incur unstable data delivery performances. Efficient data dissemination to a desired number of receivers in a vehicular ad hoc network (VANET) is a new issue and a challenging one considering the dynamic nature of VANETs. This paper presents an overview on simple and robust dissemination technique that efficiently deals with data dissemination in both dense and sparse vehicular networks. This technique divides the users in two categories and it takes three cases.
\end{abstract}

\section{General Terms}

VANETs, Data Dissemination

\section{Keywords}

Data Dissemination, techniques, ASSD

\section{INTRODUCTION}

VANET is a new technology that integrates the potentials of new generation wireless networks into vehicles. VANET focuses to offer (i) continuous connectivity for mobile users while they are on the road, which enables them to link with other users through the latter's home or office based networks and (ii) efficient wireless connection between vehicles without access to any fixed infrastructure which enables ITS. VANET is also known as inter-vehicle communication (IVC). VANET devices such as on -board units are fixed in vehicles and functions as the nodes to transmit and receive messages through wireless networks.
These devices provide drivers and passengers with the latest information on accidents, flooding, rain, traffic etc. By obtaining such information on time, drivers can make appropriate decisions and avoid misfortunes. Altering drivers about the conditions of roads, traffic and related aspects are crucial to safety and to the regulation of vehicle flow. In future vehicular networks, the requirements for communication between vehicles and the outside world will only increase. The communication protocol that is used as a standard to support wireless access in vehicular environment (WAVE) was recently defined by the ITS IEEE $802.11 \mathrm{p}$ Task Group [1]. Communication modules are expected to be embedded in vehicular entertainment systems, integrating the Microsoft Silver-light streaming technique and Microsoft Expression Encoder to deliver Smooth Streaming effect and provide users with a high-quality viewing experience. [12]

\section{OVERVIEW ON VANETS}

Vehicular ad hoc networks are raising new technologies to combine the functionality of new wireless networks with vehicles. The key workings of a VANET are wireless on board unit (OBU), the roadside unit (RSU) and the authentication server (AS). OBUs are installed in vehicles to give wireless communication capability, while RSUs are deployed on intersections or hotspots as an infrastructure to provide information or access to the internet for vehicles within their radio coverage.

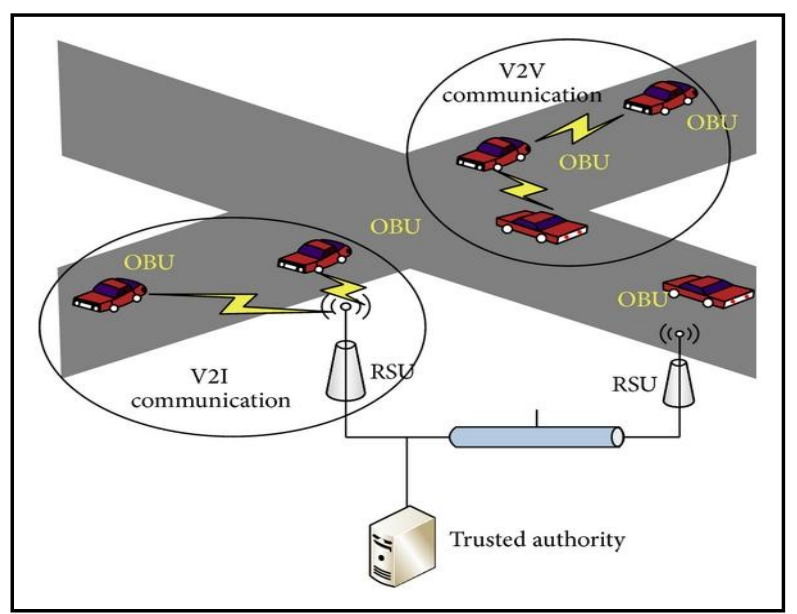

Fig1: VANETs [1] 


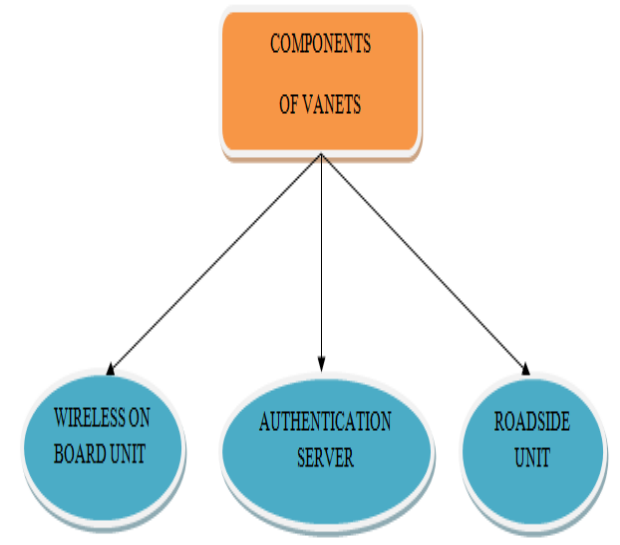

Fig 2: Components of VANETs

- Wireless On Board Unit:- On Board Units (OBU) work via GPS, the on-board odometer or tachograph, a digital map and GSM (used to authorize payment via a wireless link).

- Authentication Server:- The AS is liable for installing the secure parameters in the OBU to validate the user.

- Roadside Unit:- RSU acts similar to a wireless LAN access point and can provide communications with infrastructure. Also, if required, RSU must be able to allocate channels to OBUs.

The recent adoption of the various 802.11 wireless standards has caused a dramatic increase in the number of wireless data networks. Today, wireless LAN is highly deployed and the cost for wireless equipment is continuing to drop in price. Currently, an 802.11 adapters or access point (AP) can be purchased for the next to nothing. As a result of the high acceptance of the 802.11 standards, academia and the commercial sector are looking for other solutions for these wireless technologies. Mobile ad hoc networks (MANAT) are one area that has recently received considerable attention. One promising application of mobile AD Hoc Networks is the development of vehicular AD Hoc Networks (VANAT). The creation of Vehicular AD Hoc Networks has also spawn much interest in the rest of the world, in the German there is the Fleet Net project and in Japan the ITS project. Vehicular Ad Hoc Networks is known under a number of different terms such as inter-vehicle communication (IVC), Dedicated Short Range Communication (DSRC) or WAVE. The goal of most of these projects is to create new network algorithms or modify the existing for use in a vehicular environment. In the future vehicular AD Hoc Networks will assist the drivers of vehicles and help to create safer road by reducing the number of automobiles accidents [2].

\section{CHARACTERISTICS OF VANET}

Unique characteristics of VANETs that distinguish them from MANET are as follows:-

- High Mobility

- $\quad$ Predictable and restricted mobility patterns

- Rapid topology change

- No power constraints

- Localization

- Abundant network nodes
- Hard delay

- $\quad$ Large scale network

- Energy storage and computing

\section{DATA DISSEMINATION AND ITS TECHNIQUES}

Data Dissemination is the communication among the vehicles and roadside base station. Vehicles can access the data through $\mathrm{V} 2 \mathrm{~V}$ technique or V2I technique. These vehicles can obtain information from the roadside base station. Each vehicle communicates with nearby vehicles in a highly dynamic ad hoc networking environment through $\mathrm{V} 2 \mathrm{~V}$ communication. Data dissemination is a process of distributing data or information over distributed wireless networks, which is superset of VANET s. Data aggregation is used to reduce the number of data transmit ion in communication medium. Basically it is used to reduce the redundant data transmission .but data aggregation approach are unsuitable for the dissemination of safety related data in VANETs. This drawback is reduced with the help of Event Suppression for Safety message Dissemination (ESSMD).The ESSMD method is inspired by the principle of data aggregation i.e. lessening of redundant transmission this suppression scheme looks to restricting the number of sources that report the same event.[3][4] The data dissemination approaches in VANET network are:-

- V2I/I2V Dissemination (vehicle to infrastructural, RSU)

- V2V Dissemination

- Opportunistic dissemination

- Geographical dissemination

- Peer-to-peer dissemination

- Cluster based dissemination

i) V2I/I2V Dissemination: It consists of two type's data dissemination- push based and pull based. In push based data dissemination, the data can be efficiently delivered from the moving vehicles or fixed base station (RSU) to another vehicle, it is mostly used in the traffic conditions, e-advertisement. While in pull based data dissemination any vehicle is enabled to query information about specific location or target it is form of request and respond type model. Mainly used in enquiry about the parking lot, nearby coffee shop basically non-popular data which user specific [3][5].

ii) V2V Dissemination: In vehicle to vehicle data dissemination, data transmit to the vehicle by another vehicle. If vehicle is out of range of cluster of Base station then base station sends the data to the vehicle through another vehicle.

iii) Opportunistic Dissemination: It is data centric architecture in which applications are not bothered with transferring the data to the right place. It uses the store and forward policy. In this, routes are built dynamically.

iv) Geographical Dissemination: When continuously topology change the end to end paths are not constantly present in VANET a geographic dissemination is used in by sending the message to 
the closest node toward the destination till it reaches. Sometimes geo-casting is also used to deliver message to several nodes in geographical area [3].

v) Peer-to peer Dissemination: In Peer-to-peer dissemination, the source node stores the data in its storage device and do not send them in the network till another node asks for them. This is proposed for delay tolerant application.

vi) Cluster based Dissemination: For a better packet delivery ratio and to reduce broadcast storms, a message has to be relayed by a minimum of intermediate nodes to the destination.To do so, nodes are organized on a set of cluster in which one node or more gathers data in his cluster and send them after to the next cluster .cluster based solution provide less propagation delay and high delivery ratio with bandwidth fairness[3] [7]

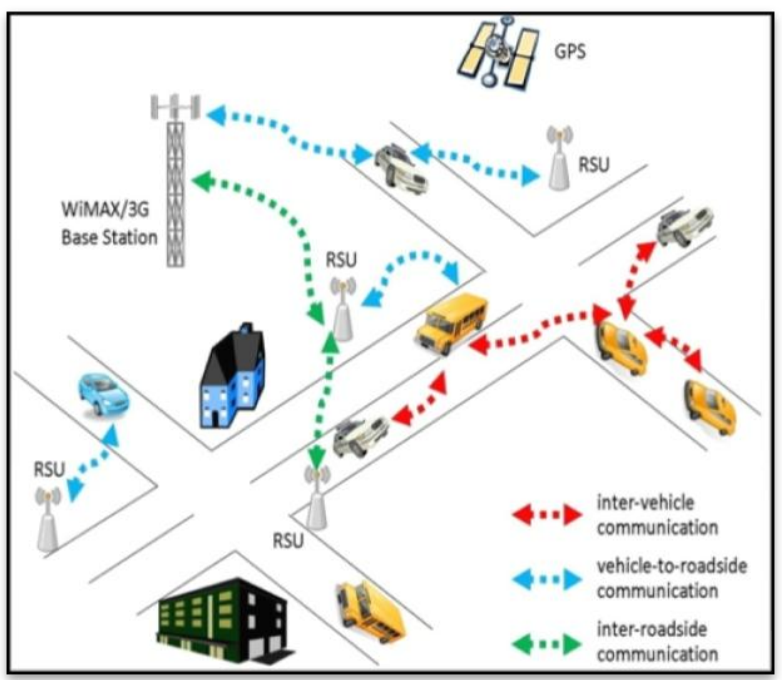

Fig 3: V2V and V2I Communication [6]

\section{CHALLENGES IN DATA DISSEMINATION}

- High Mobility and Frequent Disconnect Topology:- The challenge in VANETs the high mobility and frequently disconnected topology at different parts of metropolitan area. The traffic density is sparse in suburban areas and during the night, but the network node density is extremely high in downtown area during the rush hours in day time, which cause frequently network disconnection. There is no simple solution for disseminating data to all receivers spreading across the metropolitan.

- Data Delivery in Presence of Disconnection:- When target vehicle move closer to the roadside unit and located in densely area, disconnection is less concern. But it's major problem when different vehicles close to one another requesting the same data at the same time and moves from base station range. When a vehicle moves within the one-hop range of the roadside units, data can be delivered to the vehicle at the highest throughput. Thus a vehicle passes by the roadside unit, it is most desirable to extend the connection time between the vehicle and the roadside unit so as to disseminate more data.
- Data Distribute Over the Mesh Nodes:- For more efficient data disseminate, multiple roadside unit can be wirelessly connected together to form a mesh network infrastructure and the cooperatively disseminated data to the vehicles .Hence it become very difficult how to distribute the data over the mesh nodes.

- Data Pass through Different Structures is Difficult: - Many existing structure such as, clustering, grid and tree in these data dissemination are extremely tough to set up and maintain because of network diversity and mobility. The network can be sparse at some region. Which may result in frequent network disconnection and network partition, if node density is high, the conventional broadcast based mechanism for data dissemination may lead to broadcast storm.

\section{MULTIMEDIA STREAMING FOR VEHICULAR NETWORKS}

With advanced network technology, people can exchange multimedia information through the Internet, including vehicular networks, which are expected to become more popular in the future. Through vehicular networks, vehicles can interchange real time information in order to avoid accidents, and transmit digital media using the P2P technique. At present, a lot of research is working on vehicular packet transmission and clustering to improve the performance of the overall system. Qadri presented a multisource video streaming mechanism for VANETs and ensured its practicality and feasibility [8]. Qian provided the basic design selections and rules for unmanned vehicular video streaming [9], and Gotta proposed a technique called Smart Mode to increase the Quality of Experience (QoE) and decrease the waste of bandwidth for satellite video streaming transmission in urban vehicular environments [10]. These applications show that video streaming in the scope of vehicular networks is both realistic and of practical interest. Multimedia streaming can be serving either on demand or live. In the case of live streaming, such as interactive video conferencing, the compressed media source needs be promptly transmitted to multimedia players through servers and networks. In on-demand streaming, all compressed media source files firstly saved in the database, and then the chosen files are transmitted to the client from the database whenever the services are requested. Nowadays, streaming techniques can be categorized into three types.

The first type is sending multimedia data to the client through traditional Web servers. This type is also called HTTP Streaming, because the web server works on Hyper Text Transfer Protocol (HTTP).

The second type is playing multimedia files through independent streaming servers, a technique which is oftenly known as True Streaming. True Streaming works on Real Time Protocol (RTP), it is also known as RTP Streaming. RTP, which is used for real time streaming and is similar to HTTP and FTP, involves sending a unidirectional data stream to players, where the data will be played right away if the bandwidth is sufficient.

The third streaming type is Clientless Streaming. In this streaming type, users play stream files with Java player, which was not built into the client-side originally. Thus, Java player will be delivered to the client only when it is requested. 
The goal of this streaming technique is "Streaming media at anywhere, anytime, anyplace and any device". However, transmission bandwidth changes in some environments, results in failure in transmission of data or wastage of bandwidth

In the vehicular network environment, various traffic conditions and the client's rapid movement make multimedia transmission more complicated. Yoon proposed a mobility profile aided files downloading service (MoPADS) for vehicular networks [11]. MoPADS uses driver's mobility records to help data transmission. More specifically, MoPADS predicts user's routes, moving speeds, and arrival time. It also establishes a mesh network, in which each macro BS is connected with a fiber backbone network and several micro BSs. The advantage of MoPADS is that the cost on BS deployment will be decreased because micro BSs no longer connect to the backbone network. However, service interruption may occur while a vehicle moves out of the coverage of a BS, since multiple route choices will make source transmission more complicated [12]. For vehicle-tovehicle $(\mathrm{V} 2 \mathrm{~V})$ data transmission this approach does not work. Sukuvaara considered both V2V and vehicle- toinfrastructure (V2I) communications to build a wireless traffic service platform (WTSP) which consists of a central traffic service server, BSs, and moving vehicles [13]. This platform provides information that requires less bandwidth, such as accident warnings and local weather reports, so streaming service was not working properly, and even in some cases it was not available.

Karimi proposed a novel LTE-based solution to support high throughput and continuous multimedia services for high speed train passengers [14].Approach was based on cell array architecture; they organized the cells along a railway, together with femto cell services. Karimi assumed that the driving direction and speed of a high-speed train are known in advance, the cell array predicts the upcoming LTE cells in service effectively, and thus enables a seamless handover. However, the driving direction and the velocity of the high speed train are fixed, and this cannot be applied to the situation where driver's destinations and velocities are unknown, as in ordinary vehicular network environments.

Annese presented an improved version of the routing protocol for vehicular networks, named smart-windows BATMAN, to allow vehicles to connect to the different roadside mesh nodes in a seamless manner. [15]

Wang [considered the blind zone and the driving direction for Cooperative VANETs, and designed a network-codingbased packet-exchange scheme that showed how to schedule the broadcast and relay process. However, relative velocity is high, the more packet error ratio the result showed. Moreover, situations where there were no relay nodes, such as the cases where the gap between two vehicles is too long or no vehicles are behind the vehicle that requests the relay service, were not considered [16].

\section{AN ADAPTIVE MULTIMEDIA STREAMING DISSEMINATION SYSTEM FOR VEHICULAR NETWORKS}

An adaptive seamless streaming dissemination system is proposed to tackle the above mentioned challenges. The adaptive streaming system prefetched stream data for each user, and stores them temporarily at the local server, based on the current situation of the users and the environments that they are located at. Several techniques such as stream data prefetching, stream data forwarding, and adaptive dynamic decoding were utilized for enhancing the adaptability of different users and environments and achieving the best transmission efficiency. Considering the uneven deployment of roadside base stations (BS) and vehicles, the bandwidth reservation mechanism for premium users was proposed to ensure the QoS of stream data which premium users received. Fuzzy logic inference system is used to determine if a BS or a vehicle can be chosen to transfer stream data for users or not. Fuzzy logic technique is adopted in this scheme because it has been efficiently used to solve various problems regarding VANETs, as well as some problems related to resource assignment [17][18], and is thus a powerful tool for controlling processes that are difficult to model and linearize.

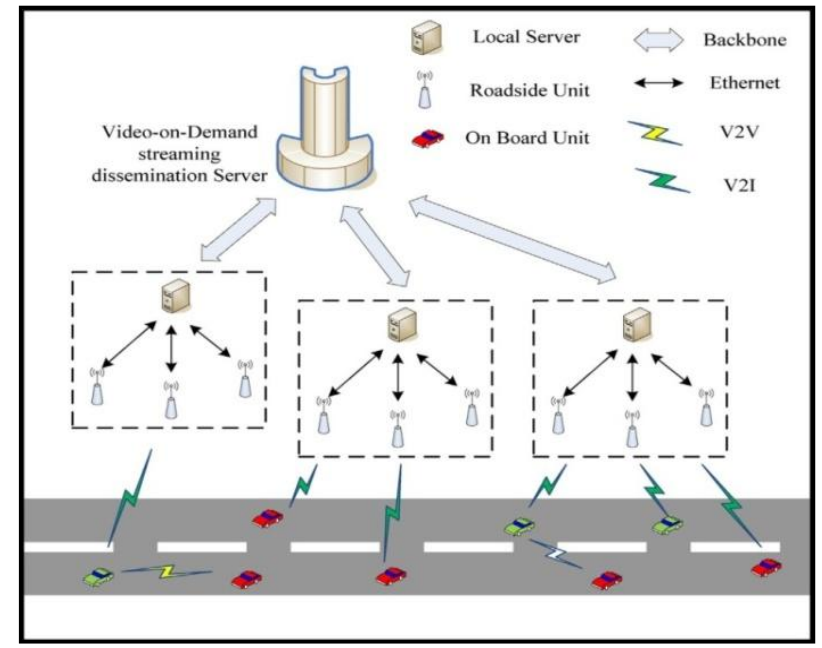

Fig. 4: Scenario of the proposed multimedia streaming dissemination system [12]

In this scheme, firstly the simulated roadmap is divided into certain parts and the scenario of the multimedia streaming dissemination system is illustrated in Fig 4. This scheme is based upon hierarchical framework, so a video on demand streaming dissemination server collects video and some streaming data buffers are distributed at each local server. Each road section is handled by a local server, whereas an upper-level server at a higher hierarchical level is responsible for a section with a larger range, which is made up of various nearby road sections. Depends upon an individual user's current status, the VoD streaming dissemination server determines the amount of frames that each local server needs to prefetch and then saves data in the local server so that congestion can be effectively avoided. It is this architecture which shows the scalability of the used scheme. The VoD stream dissemination server and the local server are connected with a backbone high-speed network. Because the distance from any local server to the roadside BSs is so close, the wired network is established to connect the local server to the roadside BSs. The connections between local servers and the roadside BSs are through wired networks, and not a wireless network, any problem will not occur between local servers and the roadside BSs during the process of prefetching the streaming data.

In this scheme, users are classified into two types:

i) Premium users

ii) Free users. 
This scheme provides free streaming service for free users but with limited bandwidth. If there is the shortage of bandwidth, then the quality of the streaming data will be less and compression ratio, frames per second for the free users will be degraded in order to provide more bandwidth to meet the minimum bandwidth requirements for the premium users who have higher priority. The free users cannot only obtain free seamless streaming service, but they also help premium users to keep accessing Internet resources without interruption and degradation of the service [12]. Hence, a win-win situation could be achieved in this scheme. When a vehicle enters the range of a roadside BS, the streaming data will be forwarded to the vehicle through the roadside BS, as illustrated by the V2I symbol in Fig. 4 and if the vehicles are outside of the communication coverage of any BS then they will require the streaming data to be forwarded through nearby vehicles, as illustrated by the V2V symbol in Fig. 4. Flow of operations of an adaptive multimedia streaming dissemination system for vehicular networks is shown in Fig. 5. For maintaining the reliable quality of the video for the viewers, the whole scenario is divided into three cases in an adaptive multimedia streaming dissemination scheme.

1) The density of roadside BSs and vehicle distribution are both high

2) The density of roadside BSs is sparse but density of vehicles is dense

3) The density of roadsides BSs and vehicle distributions are both low.

With the received data, the system determines each user's case to make sure the performance of video streaming service is maintained at a satisfactory level. As shown in the right portion of Fig. 6, a user first downloads and plays stream data from a roadside BS simultaneously.

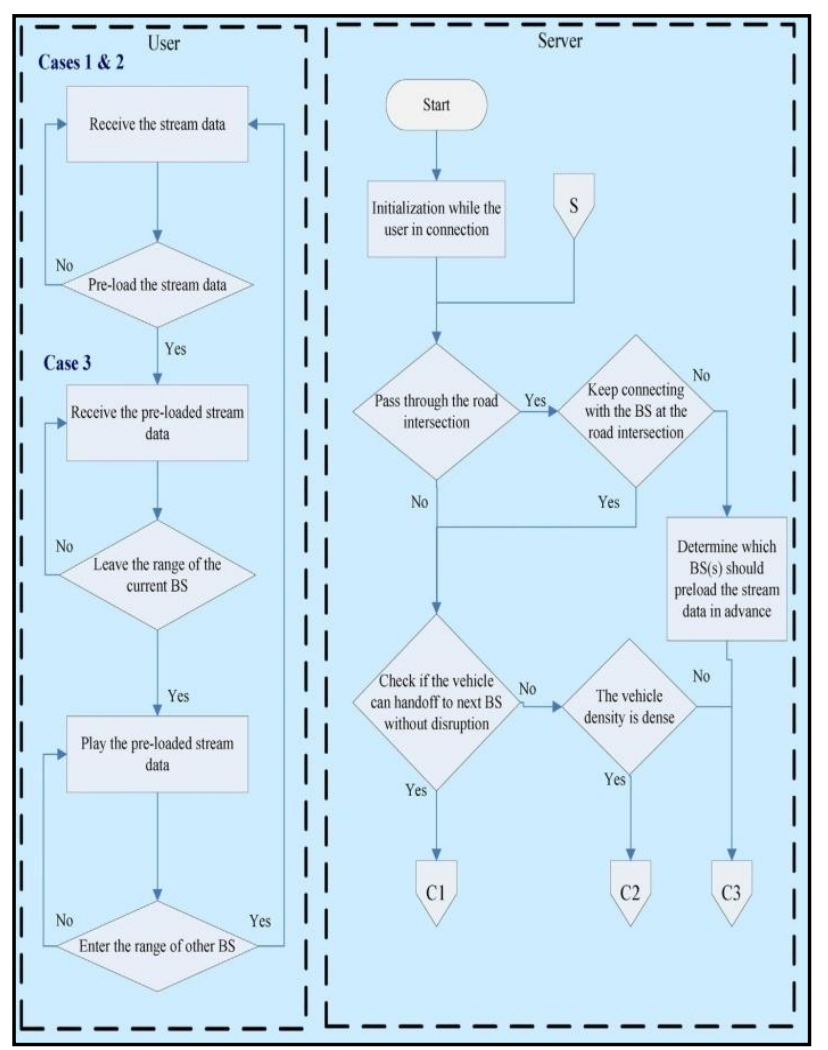

Fig.5: Flow of system operations [12].
In Cases 1 and 2, it is not necessary to preload the stream data in advance. Even there are few roadside BSs in Case 2, the user can still receive the stream data through other vehicles acting as forwarding nodes. When the number of vehicles and roadside BSs are insufficient to maintain the live streaming service, a preloading mechanism will be used to make sure that the users, especially the premium users, can still enjoy high viewing quality. A user will keep receiving the preloaded stream data until his or her vehicle leaves the range of the current BS, and then plays the preloaded stream data to meet a seamless live stream service. When the user enters into the range of another roadside BS, the user will start receiving the stream data from the BS again [12]. The above-mentioned operations of the server are shown in the right part of Fig. 5.

Case 1: The density of roadside BSs and vehicle distribution are both high

We know that the density of roadside BSs and vehicles distribution are both high in populated urban areas. As we know that the number of roadside BSs is much higher than in the suburbs or along suburban roads, the number of users accessing the Internet also increases at the same time, and this may a result in shortage of bandwidth and the problem of congestion. So in this scheme it considered that the possibility of the BSs having an unconnected status. Assume that the green vehicle illustrated in Fig. 6 is exiting the cluster of the current roadside BS, and it will pass through an intersection on its left shortly.

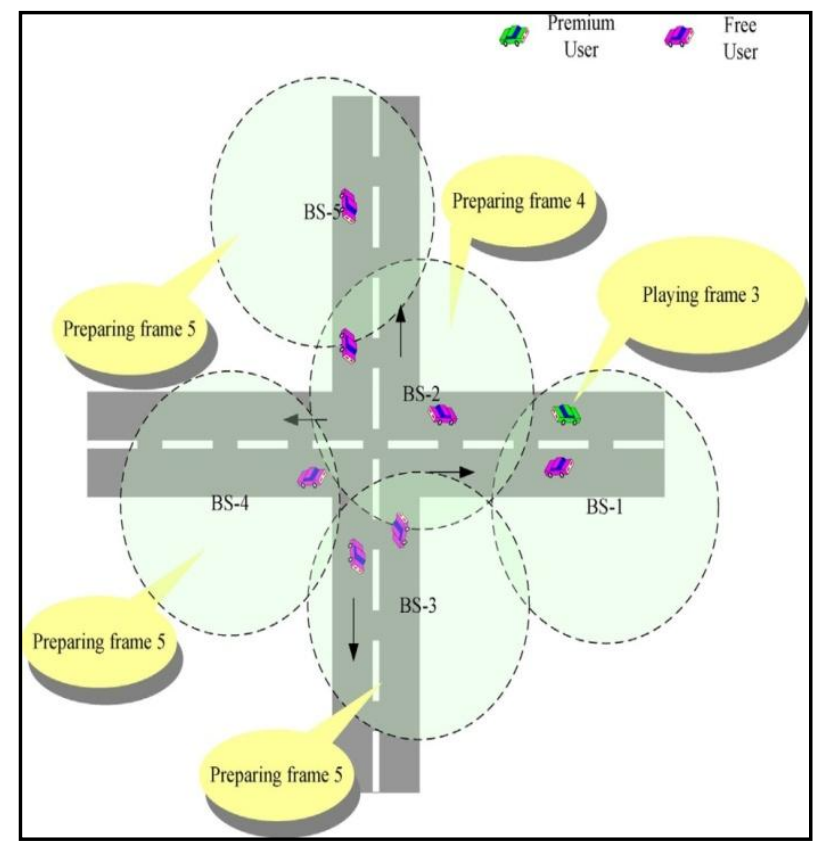

F ig 6: The scenario of a vehicle passing through a road intersection [12].

It is possible for the vehicle to leave the range of BS-2 and enter the coverage of BS-3, BS-4, or BS-5 after the vehicle passes through the intersection. The problem is the server does not predict that in which direction the vehicle may drive. Let us take Fig. 6 as an example. When the green vehicle is accessing the Frame 3, Frame 4 has been preloaded by the BS-2. In this scheme, we allow Frame 5 to be preloaded by BS-3, BS-4, and BS- 5 because the user will enter the intersection area, and server have no idea in which direction the vehicle will go. This method will repeat itself and preload the streaming data at different roadside BSs, the 
time to determine the direction is very less and the amount of repeated preload streaming data is not very large. So, the extra overhead is acceptable in this scheme. As shown in the right portion of Fig. 5, when a vehicle is not going to pass through an intersection, and the vehicle can handoff to the neighboring BS after the vehicle disconnects with the current $\mathrm{BS}$, the system will take the actions after label $\mathrm{C} 1$, which is also the initial stage shown in Fig. 7. And if a vehicle will pass through an intersection and the vehicle can handoff to a neighboring BS, the system will take the actions after label $\mathrm{C} 1$ as well. In both the situations, the system will check if there is enough free bandwidth at the neighboring BS. As illustrated in Fig. 7, the needed bandwidth to prepare transferring streaming data for the vehicle will be reserved if the system gets a positive acknowledgment from the neighboring BS. If the system detects that the vehicle cannot handoff to any neighboring BS after the vehicle is outside of the coverage of the current BS, the system will take the actions after label $\mathrm{C} 2$, which illustrated in Fig. 8. In this situation, the vehicle will try to find a route to the next available BS through Vehicle to Vehicle communications. The requesting vehicle firstly broadcasts a packet to find a route to the destination. Each vehicle on the way to the destination is asked to reply to the source vehicle as to whether or not it is appropriate to act as a relay vehicle for forwarding the streaming data for the requesting vehicle [12].

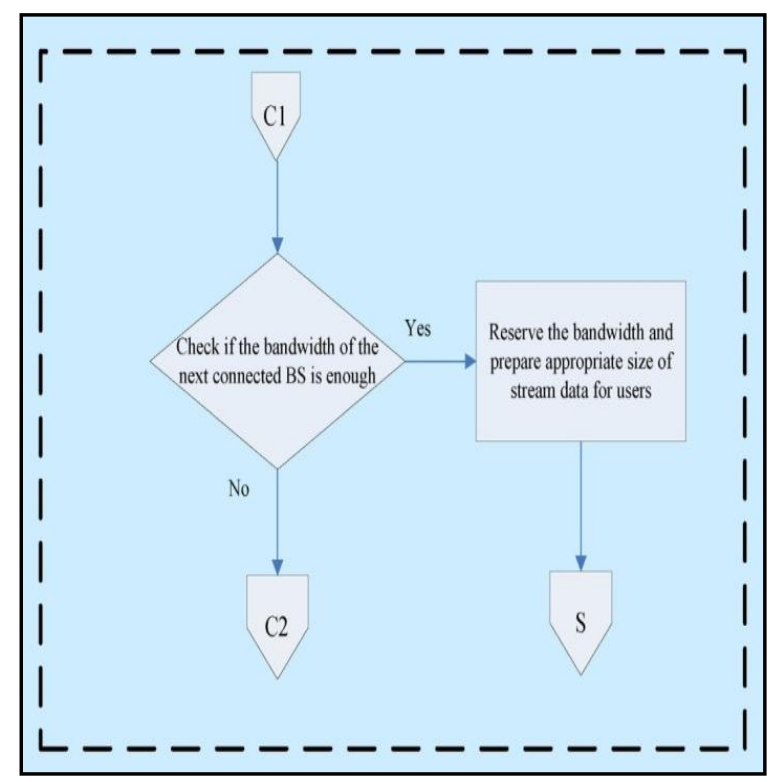

Fig. 7: The actions after Label $\mathbf{C 1}$

Here, a fuzzy logic inference system is used to determine the finest transferred route by selecting the most suitable relay vehicle(s) to forward the streaming data for the requesting vehicle. After the broadcast packet received by the closest base station(BS) on the route to the destination, a routing path is then maintained by selecting the most finest nodes to forward the packets along the way to the destination or the closest base station. In this scheme, the radius of the coverage area of each relay vehicle is assumed to be identical to the length of a road segment [12]. Now we will find the optimal path to send the data by using fuzzy logic interference system. As we discussed in above section that, the routing path is maintained through Vehicle to Vehicle communications to maintain this path system will select the most appropriate intermediate nodes along the way to next roadside BS, assuming the radius of the coverage area of each vehicle is identical to the length of a road segment. The optimal transferred route, is determined by calculating an appropriateness level for each candidate relay vehicle. The system will first calculate an appropriateness level of all vehicles among the requesting vehicle and the next BS with enough bandwidth for the requesting vehicle. The distribution of vehicles is dynamic in this scheme, not only one route would be selected as the transferred path for the requesting vehicle, and a routing path composed of neighboring vehicles with the smallest appropriateness levels along the way from the requesting vehicle to the next available BS will be recommended by the system as the transferred route for relaying streaming data.

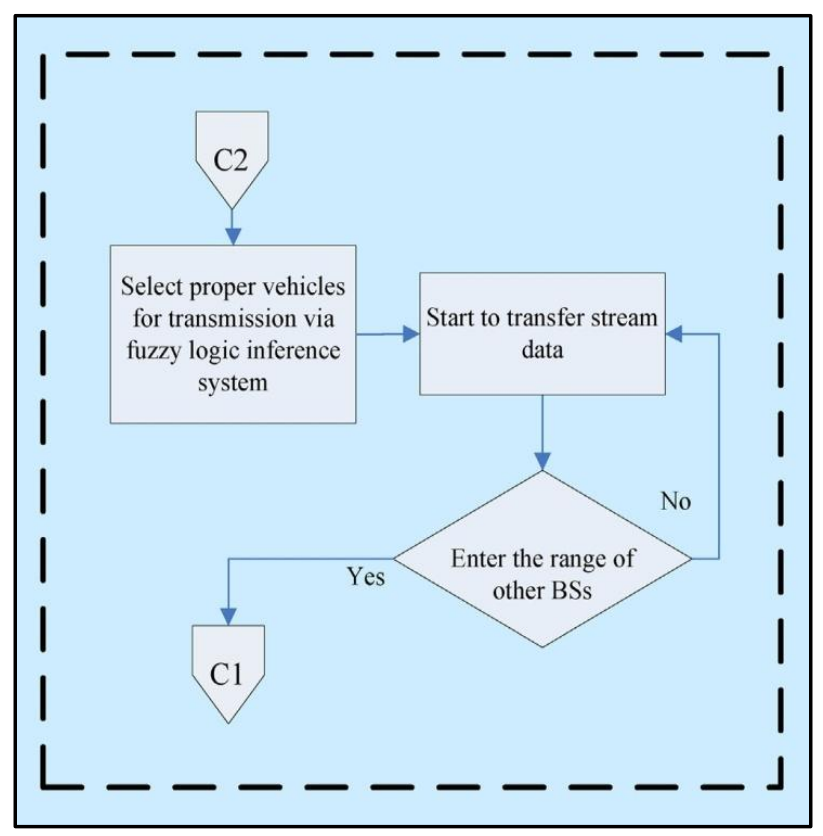

Fig. 8: The actions after Label C2

To find out the finest intermediate nodes for maintain the path, compare the feedback values sent back by the vehicles distributed at the road segment.

$$
\text { inode }={ }_{i}^{\max } f l i(.) \text {, }
$$

fli(.) represents the output generated by the fuzzy logic interference system at the vehicle indexed by $\mathrm{i}$.

Fig.9 shows the input-output elements to determine the appropriateness level of a candidate relay vehicle. There are three input parameters used in the fuzzy logic inference system,

i. Minimum Bandwidth Required:- To satisfy the streaming service for the requesting vehicle

ii. Estimated Remaining Connection Time:-It is the estimation of time between the candidate relay vehicle and its neighbor vehicles. This can be easily calculated by a simple computation based on the distance between the two vehicles and their relative speed. Moreover, the selected relay vehicles may have similar characteristics, with the same driving direction and the same driving speed as the requesting vehicle, which can provide stability while transferring streaming data.

iii. Currently Used Bandwidth:-This is the third input which determines the bandwidth currently being used by the candidate relay vehicle. 
Moreover, insufficient bandwidth or number of free users and premium users in connection will affect the Quality of service of playing stream data, and service disruptions can occur. The basic functionality of each component in the fuzzy logic interference system is described as follows:-

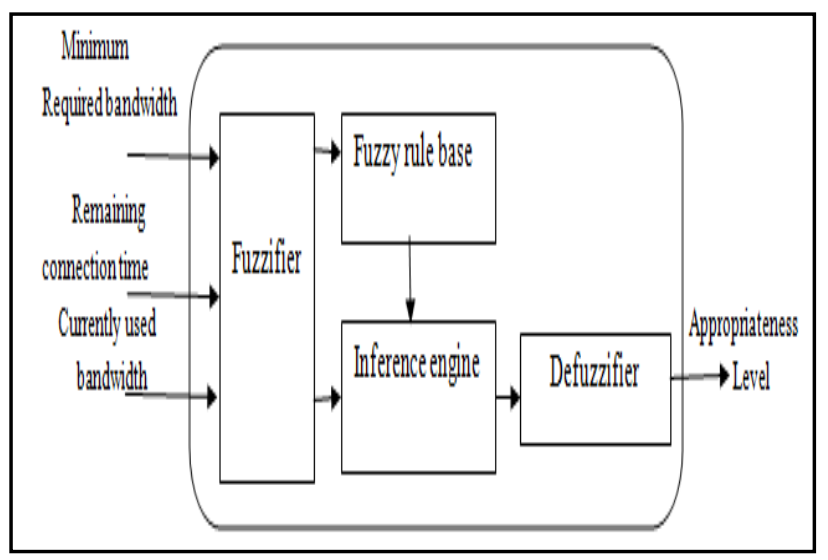

Fig. 9: Architecture of fuzzy logic inference system algorithm used in Fig. 8[12].

i) Fuzzifier: The fuzzifier performs the fuzzification function that converts three inputs into suitable linguistic values which are needed for the calculations by the inference engine.

ii) Fuzzy rule base: The fuzzy rule base is composed of a set of linguistic control rules and the attendant control goals.

iii) Inference Engine: The inference engine simulates human decision-making, based on the fuzzy control rules and the related input linguistic parameters.

iv) Defuzzifier: The defuzzifier acquires the aggregated linguistic values from the inferred fuzzy control action, and generates a non-fuzzy control output, which represents the predicted priority.

Rule middle $\mu$ low $\mu$ low min $\mu$ middle middle

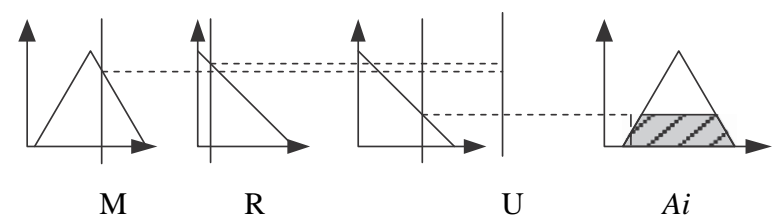

Fig. 10: The reasoning procedure for the Tsukamoto defuzzification method[12].

Input $\mathrm{M}$ denotes minimum bandwidth required for satisfying the real time traffic $\mathrm{R}$ denotes an expected remaining connection time between the vehicle indexed by $\mathrm{i}$ and its neighbor vehicles $U$ denotes the band- width currently used at the vehicle indexed by i. $\mu$ denotes the degree of membership.

Fig. 10 shows the defuzzification stage of the fuzzy logic inference system. The fuzzy linguistic variables "Small", "Middle" and "High" give different required minimum bandwidth measures in the membership function for the input M. Similarly, three linguistic term sets, "Short", "Medium" and "Long" are used for R, and "Small", "Middle" and "High" are used for U. The output parameter of the inference engine, $\mathrm{A} i$, the appropriateness of the vehicle as an intermediate relay node on the transferred path, is defined as the estimated link quality when the vehicle is chosen as the intermediate node. The range of the output falls between 0 and 1 . There are 27 inference rules generated in the Mamdani fuzzy inference system. The non-fuzzy output of the defuzzifier can then be expressed as the weighted average of each rule's output after the Tsukamoto defuzzification method is applied:

$$
A L=\frac{\sum_{j=1}^{27} A j \cdot w j}{\sum_{j=1}^{27} w j}
$$

Where $A j$ denotes the output of the $\mathrm{j}^{\text {th }}$ rule induced by the firing strength $j$.

Case 2: The density of roadside BSs is sparse but density of vehicles is dense

Let us take Fig. 11 as an example, where the density of roadside BSs are sparse and users might not communicate with any BS, and the system will instead allow a vehicle to maintain the streaming service by using neighboring vehicle(s) to transfer the stream data, with priority given to premium users that enjoy a minimum QoS guarantee. The system will request neighboring vehicles to act as transmission nodes to provide the stream data along the suggested transferred path. As shown in Fig. 11, the premium user (green car) in the intersection is out of the range of any roadside BS, the user can still enjoy the viewing quality of streaming data through the nearby vehicles, which are all free users in this example.

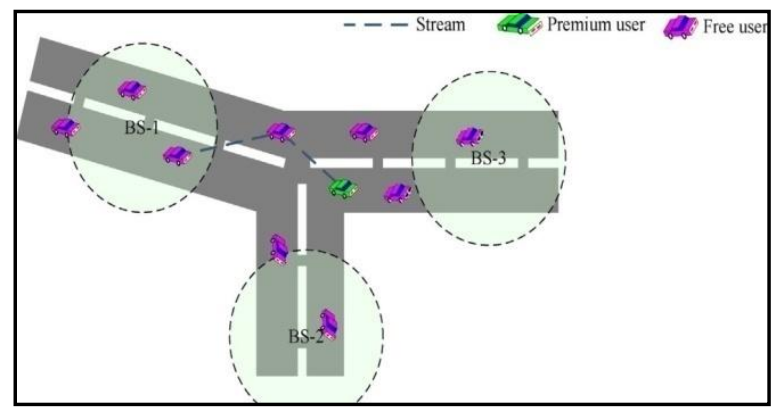

Fig. 11: An example that shows roadside BSs are sparse but the vehicle density is high.

Right portion in Fig. 5 defines the operations of the system for Case 2. In case 2, the distance between the current BS and the next BS is large, and the vehicle density is high. While entering into label $\mathrm{C} 2$ as shown in Fig. 8, the system firstly use the fuzzy logic inference system to select suitable vehicles for transferring streaming data. Next, the selected vehicles will be asked to provide bandwidth for the requesting vehicle until it enters the transmission range of any roadside BS. The system will then turn to the operations after label $\mathrm{C} 1$ to determine if the next BS is appropriate for forwarding streaming data, as shown in Fig. 7.

Case 3: The densities of roadsides BSs and vehicle distributions are both low

Let us take an example is shown in Fig. 12, where we can see that there is a large gap between BS-2 and BS-3 will result in a service disruption for the vehicle that is requesting for the streaming service. To provide the streaming service, the server will ask BS-1 and BS-2 to activate the preloading mechanism to download the compressed stream data in advance. Now let's check the right portion of Fig. 5 to start the operations for Case 3. If the system detects that the requested vehicle's streaming service cannot connect to any BS or relay vehicles, as shown in the example in Fig. 12, the 
system will take the actions after label $\mathrm{C} 3$, which is also the starting stage in Fig. 13.

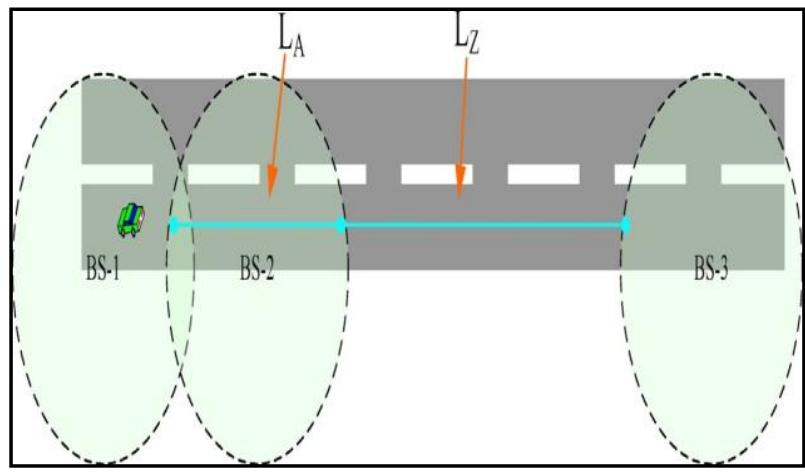

Fig. 12: An example that shows the densities of roadsides BSs and vehicle distributions are both low

When the user doesn't receive the streaming service neither from any roadside BS, nor from other nearby vehicles available to act as relay nodes in the near future, the server will activate the pre-loading mechanism at the BSs so that the user vehicle can remain connected. The compression ratio of the stream data can be estimated based on the current speed of the vehicle and the distance that the vehicle will remain connected. The system will continuously enjoy the current stream data and preload the necessary streaming data in advance, according to the estimated compression ratio. Once the user enters the cluster of any roadside BS, such as BS-3 in the example shown in Fig. 12, the server will classify the user into Case 1 and prepare the connection with the BS

Take Fig. 12 as an example again, a vehicle is driving on the road section $L_{A}$ and is going to pass through the intersection of BS-1 and BS-2. The server is aware that this vehicle will be outside of the coverage of all the BSs in the following road section $\mathrm{L}_{\mathrm{Z}}$. Here the two parameters, $\mathrm{L}_{\mathrm{A}}$ and $\mathrm{L}_{\mathrm{Z}}$, which represent the road section with connection and the road section outside of the transmission range of any BS, are used to calculate the compression ratio of the stream data. Assume that the vehicle moves with constant velocity, and the driving times on $\mathrm{LA}$ and $\mathrm{L}_{\mathrm{Z}}$, are directly proportional to the distance. So, we can compute how much bandwidth we should reserve in advance while driving on $\mathrm{LZ}$ and further calculate the appropriate compression level of the stream data to avoid the shortage of bandwidth in the following road section where the vehicle will be without connection to any BS

\section{Assume,}

i. a $\mathrm{LZ}$ represent the required bandwidth reserved in advance on the road section $\mathrm{L}_{Z}$, where the vehicle is outside of the transmission range of any BS

ii. $a_{L Z}$ denotes the allocated bandwidth while the vehicle is on the road section $\mathrm{L}_{\mathrm{A}}$, during its connection with BS-2, as shown in Fig. 13. The determination of a $\mathrm{L}_{Z}$ and $\mathrm{L}_{\mathrm{A}}$ can be expressed as follows:

- where i represent the service level of the stream data

- $\quad$ ai the required bandwidth on the road section LA

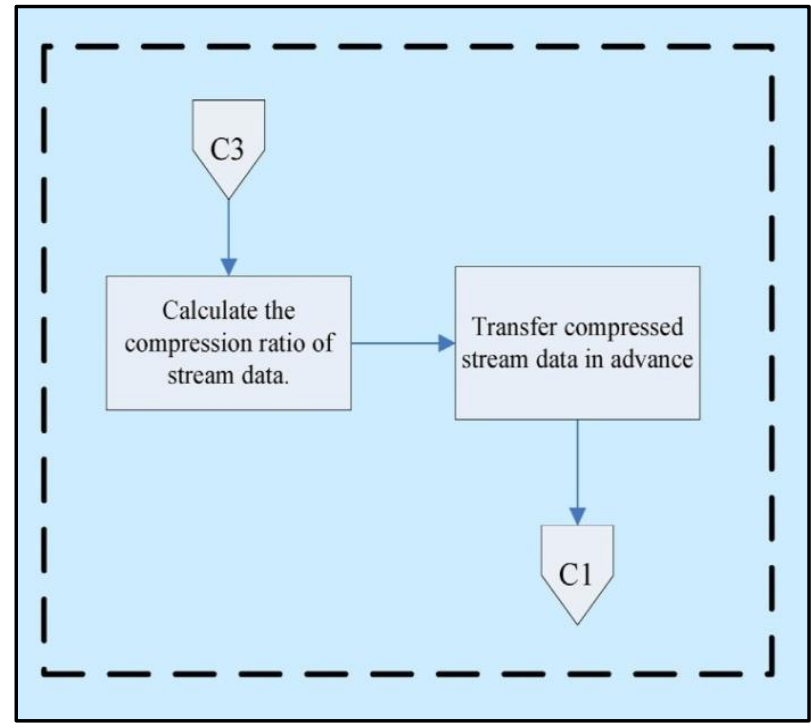

Fig. 13. The actions after Label C3 [12]

Notably, the service level of the stream data and its corresponding video resolution quality used in this scheme are listed in Table 1.

Notably, this scheme can adjust $a_{L_{A}}$ according to the ratio of the $\mathrm{L}_{\mathrm{A}}$ and $\mathrm{L}_{\mathrm{Z}}$. If the ratio $a_{i} \times \frac{L_{A}}{L_{A}+L_{Z}}$ is between $a_{k+1}$ and $a_{k}$ as specified in the third column in Table 1, this scheme selects the lower bound of the resolution, $a_{k+1}$ to prevent the shortage of bandwidth and the possibility of service disruption. As a result, if the disconnected road section Lz is not too long, then this scheme can still keep acceptable video quality with a better resolution. Else, a degraded service level of stream data will be provided to users to avoid service disruption.

$a_{L_{A}}= \begin{cases}a_{n}, & \text { if } a_{i} \times \frac{L_{A}}{L_{A}+L_{z}}<a_{n} \\ a_{k+1}, & \text { if } a_{k+1} \leq \frac{L_{A}}{L_{A}+L_{z}}<a_{n}\end{cases}$

Table 1: The service level of the stream data and its corresponding video resolution quality [12]

\begin{tabular}{|l|l|l|}
\hline $\begin{array}{l}\text { Service level } \\
\text { of stream } \\
\text { data }\end{array}$ & $\begin{array}{l}\text { Corresponding } \\
\text { video resolution } \\
\text { quality }\end{array}$ & $\begin{array}{l}\text { Required } \\
\text { bandwidth of each } \\
\text { video }(\mathbf{k b} / \mathbf{s})\end{array}$ \\
\hline $\mathrm{n}=1$ & $1920 \times 1080$ & 500 \\
\hline $\mathrm{n}=2$ & $1280 \times 720$ & 200 \\
\hline $\mathrm{n}=3$ & $848 \times 480$ & 100 \\
\hline $\mathrm{n}=4$ & $640 \times 480$ & 50 \\
\hline
\end{tabular}

Table 2: Simulation parameters [12]

\begin{tabular}{|c|c|}
\hline Parameter & Value \\
\hline Simulation area & $10 \mathrm{~km} \times 10 \mathrm{~km}$ \\
\hline Moving speed of vehicles & $40 \mathrm{~km}-80 \mathrm{~km} / \mathrm{h}$ \\
\hline Type of wireless network & Wi-Fi \\
\hline
\end{tabular}




\begin{tabular}{|c|c|}
\hline Transmission range & $80 \mathrm{~m}$ \\
\hline $\begin{array}{c}\text { Required bandwidth of } \\
\text { videos }\end{array}$ & $500 \mathrm{~kb} / 200 \mathrm{~kb} / 100 \mathrm{~kb} / 50 \mathrm{~kb}$ \\
\hline
\end{tabular}

\section{SIMULATION RESULT}

In this scheme a series of simulations ran to verify the feasibility and effectiveness of an adaptive seamless streaming and dissemination (ASSD) system. The simulation parameters are shown in Table 2 . This scheme randomly simulated a street map where the mobility pattern was generated by the Vanet MobiSim simulator, as shown in Fig. 6 , the half-side width of which is $5 \mathrm{~m}$, with bidirectional traffics streams. This scheme assumed that there are

i) 20 vehicles pulling into a broadcast zone

ii) 5 vehicles pulling into a blind zone.

iii) The distance between any two adjacent vehicles is about $20 \mathrm{~m}$

iv) The average speed of thevehicles in the simulation is $60 \mathrm{~km} / \mathrm{h}$ with at most $\pm 5-\mathrm{km} / \mathrm{h}$ deviation.

v) This scheme assume that BSs broadcast messages every $100 \mathrm{~ms}$

vi) The packet lengths are equal to $500 \mathrm{~kb}, 200 \mathrm{~kb}$, $100 \mathrm{~kb}$, and $50 \mathrm{~kb}$ respectively.

vii) The bandwidth of the broadcast channel is 10 $\mathrm{MHz}$,

viii) The transmission range between relay nodes is 80 $\mathrm{m}$.

Notably, Mat lab tools are used to generate the network scenario and traffic model addressed in this scheme. The resolution quality and the required bandwidth for each video used in this simulation are listed in Table 1.

Three representative approaches Mobility profile Aided File Downloading Service (MoPADS) Wireless Traffic Service Platform (WTSP), and the Network Coding Based Scheme (NCBS), are compared this scheme. MoPADS is also an intelligent transportation system that combines the cellular infrastructure and ad hoc distribution network. It uses vehicular mobility profiles not only to provide quality yet affordable services, but also to predict the vehicle's possible route, velocity, and the required time to get to some specific place in the future[11]. WTSP considers two types of communications, including vehicle-to-vehicle and vehicle-to-infrastructure, to construct a wireless service platform based on the traffic service central unit, the base station network with traffic service base stations, and mobile end users with ad-hoc connectivity and backbone network connectivity. It provides real-time service to mobile users with less bandwidth requirements [20]. NCBS realized the seamless information dissemination associated with V2I and $\mathrm{V} 2 \mathrm{~V}$ communications by taking into account the interruption in the transmission of information and message loss when vehicles pass through broadcast and blind zones [24]. The results showed that NCBS offered Internet streaming service with lower packet error ratio and higher throughput and this scheme makes use of free users to relay streaming service for premium users if needed, this scheme evaluated the performance for both types of users. The notation ASSD-P is used for the premium users, and ASSD-F is used for free users. Adopted performance metrics for this work are:-. i) Throughput: - Throughput represents overall multimedia data transmission capacity, which includes the data received from the adaptive streaming server and the data shared between users through the BSs bandwidth sharing mechanism.

ii) Packet loss ratio: - Packet loss ratio is used for the percentage of packets that get lost, producing errors in the transmission process.

iii) Service disruption time:-Service disruption time is defined as the period of time during which the vehicle cannot maintain active connection with the current BS until it connects with a new serving BS link and restarts sending and receiving packets.

Fig. 14 shows the packet loss ratios under the condition of different numbers of vehicles among the four schemes. ASSD scheme achieves better performance in terms of packet loss ratio than the other systems and the reason is that this scheme ensures the QoS in different traffic conditions. For example, if the density of vehicle distribution is low, the number of relay vehicles is also comparatively low, and then this scheme uses the prefetching mechanism and this scheme can avoid the problems of transmitted streaming data interruption and delay. From the results, it can observe that the packet loss ratio is slowly increasing. That is because the number of vehicles is increasing and limited bandwidth is being accessed by more users. However, this scheme involves using portions of free user's bandwidth to help premium users in packet transmission, and ASSD scheme also lowers free users quality in terms of compression and frame per second (fps) to this end. The result shows that the premium users in ASSD scheme achieves the best performance in terms of the packet loss ratio, compared to the other systems, and the free users in this scheme also experience better performance than the three compared methods.

In the case of MoPADS, only a few server stations are connected to the backbone through high-bandwidth wires, and the other small BSs access the networks by sharing the bandwidth of large BSs. When the traffic flow increases, a shortage of bandwidth will occur, and thus the packet loss ratio will increase. As for WTSP, it provides real-time service with a lower requirement of bandwidth. The packet loss becomes higher when the bandwidth demand of the realtime service gets larger. WTSP can alleviate the deterioration of the packet loss ratio by adopting vehicle-to-vehicle communication to transfer stream data when the density of vehicles is high. NCBS allows the nearby vehicles to forward the data for the vehicle requesting streaming service, this scheme only considers the fairness of each vehicle as a relay node. Thus, the performance in terms of packet loss ratio for NCBS is still slightly worse than for ASSD scheme, which addresses more considerations in its implementation. As shown in Fig. 15, ASSD scheme performs best in terms of service disruption time. ASSD scheme will be well controlled, since this scheme considered the distribution of vehicles and BSs. If the density of vehicles is high, the system will transfer stream data through selected vehicles according to the most suitable distance and velocity among the vehicles. But if the density of vehicles is too low to allow for transfer of the data, the prefetching mechanism will use, to provide uninterrupted transferring of stream data to maintain the QoS. Notably, the slopes, including ASSD-P and ASSD-F, moderately increase with the increasing number of vehicles. In ASSD scheme, although the vehicles cannot receive signals from the BS while passing out of the 
coverage range of the $\mathrm{BS}$, they can use other vehicles to enjoy the continuously streaming for them. Therefore, the disruption time caused by passing through areas which are outside of the coverage of any BS can be dramatically decreased. In addition, when the number of vehicles is over 150 , the service disruption time for free users in this scheme are slightly longer than for premier users. That is because the free users need to assist the premier users in relaying streaming data and the service disruption time for the free users is thus increased more obviously than it is for the premier users.

The MoPADS scheme does not work for vehicle-to-vehicle communication, so when the vehicle drives outside the coverage of the BS, or faces the shortage of bandwidth provided by the small BS, the service disruption time will increase when the number of vehicles and the requirement of bandwidth increase. WTSP and NCBS schemes, they both allow vehicle-to-vehicle communication but have no service prefetching mechanism to transfer stream data. Accordingly, the service disruption time does not significantly degrade when the number of vehicles increases NCBS can spread forward information with as little loss as possible, while mitigating the interference to other vehicle reception of broadcast messages caused by relaying signals.

Fig. 16 shows the throughput corresponding to the conditions of different numbers of vehicles among the four schemes. Such as stream data prefetching, stream data forwarding, and adaptive dynamic decoding are applied in this model to strengthen the adaptability of different users and environments, ASSD scheme proves good in terms of maximizing data throughput, with the comparisons between the four schemes. In the MoPADS scheme, small BSs may face a shortage of bandwidth with which to transfer stream data, but once the vehicles drive back into the coverage of the small BSs, the system still can complete the packet transmission. In the WTSP scheme, although the platform it constructed based on V2V and V2I communications, it provides real-time service to mobile users with less bandwidth requirements. Hence, the mobile users with high bandwidth requirement will not be suitable for this scheme. Since vehicle speed is not taken into account in the NCBS, it is necessary to find an alternative relay node after the current relay node drops out of the communication range of the neighboring relay vehicle and its performance is thus degraded.

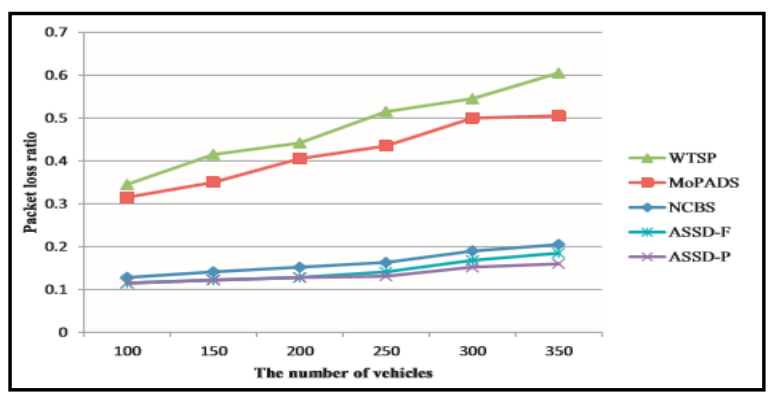

Fig. 14: Comparison of packet loss ratio among the four algorithms [12].

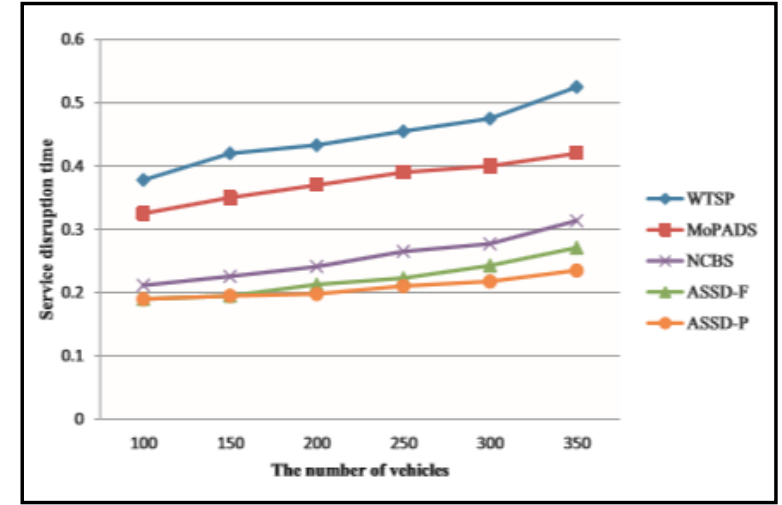

Fig. 15: Comparison of normalized service disruption time among the four algorithms [12].

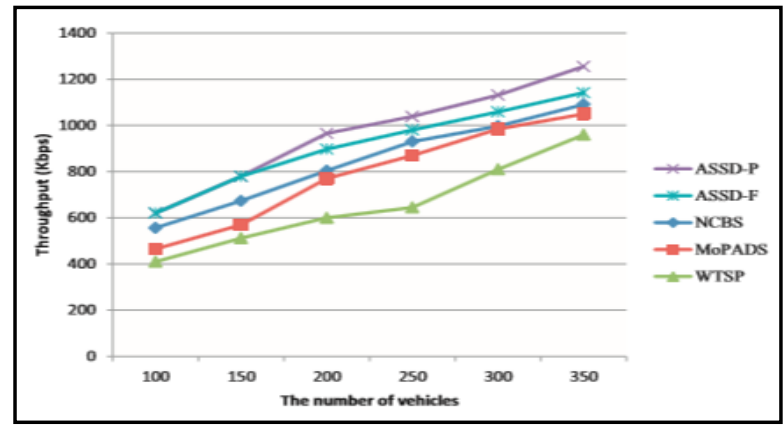

Fig. 16: Comparison of throughput among the four algorithms [12]

\section{CONCLUSION}

This paper illustrates the ASSD scheme for different users for different environment for streaming the data. Playing live stream videos in vehicles thus becomes possible via VANET and roadside BSs. However, there are some difficulties to be overcome, such as the uneven distribution of roadside BSs, the high mobility of vehicles, and the volatility of network environments. In this paper, an adaptive seamless streaming dissemination system for vehicular networks is explained to solve the above mentioned problems. Three cases are taken under some considerations to deal with different kinds of network environments according to the number of vehicles and roadside BSs. When there are few roadside BSs, the user can still receive the stream data through other vehicles which act as forwarding nodes. When the number of vehicles and roadside BSs is insufficient to maintain the live streaming service, a preloading mechanism will be used to provide the premium users so they can enjoy satisfying viewing quality, meeting the requirement for a seamless live stream service. Comparing this scheme with MoPADS and WTSP, the simulation results show that ASSD mechanism achieves better performance in terms of throughput and service disruption ratio. NCBS is slightly better than ASSD in case of throughput.

\section{REFERENCES}

[1] IEEE P802.11 - Task Group p, "IEEE P802.11p/D9,0, Draft Amendment for Wireless Access in Vehicular Environments (WAVE)", July 2009.

[2] Nathan Balon,Introduction to Vehicular Ad Hoc Networ ks and the Broadcast Storm Problem

[3] Annu Mor Research Scholar, Deptt. Of Computer Science Applications, Kurukshetra University, 
Kurukshetra,Haryana India, Study of Different Type of Data Dissemination Strategy in VANET ISSN: 23195967 ISO 9001:2008 Certified International Journal of Engineering Science and Innovative Technology (IJESIT) Volume 1, Issue 2, November 20126

[4] J.Zhao, Y.Zhang and G.Yeng" data pouring and buffering on Road: a new data dissemination paradigm for vehicular ad hoc network", IEEE transaction on vehicle technology, vol.56, 2007

[5] Pratibha Tomar, G.S tomar "state of art of data dissemination in VANETs", international journal of computer theory and engineering, vol 2, no.6 December, $20101793-8201$

[6] http://www.gta.ufrj.br/ensino/eel879/trabalhos_vf_2010 _2/lemos/introducao.html

[7] O.tongue, N. Wisitpongphan "a distributed vehicular broadcast for VANET, IEEE wireless communication in 2010

[8] N.N. Qadri, M. Fleury, M. Altaf, M. Ghanbari, Multisource video streaming in a wireless vehicular ad hoc network, IET Communications 4 (July (11)) (2010)1300-1311.

[9] H.X. Qian, J.S. Huang, L.L. Ma, The design challenges for unmanned vehicular video streaming, in: 2011 IEEE International Conference on Vehicular Electronics and Safety (ICVES), July, 2011, pp. 19-24.

[10] A. Gotta, E. Ferro, F. Potorti, Quality of Experience in Satellite video streaming transmissions in urban vehicular environment 2009, in: IWSSC International Workshop on Satellite and Space Communications, September, 2009, pp.18-22.

[11] S. Yoon, D.T. Ha, H.Q. Ngo, C. Qiao, MoPADS: a mobility profile aided file downloading service in vehicular networks, IEEE Transactions on Vehicular Technology 58 (November (9)) (2009) 5235-5246.

[12] Chenn-Jung Huang, Yu-Wu Wang , Heng-Ming Chen , Ai-Lin Cheng, Jui-Jiun Jian, Han-Wen Tsai, Jia-Jian Liao,An adaptive multimedia streaming dissemination system for vehicular networks Applied Soft Computing 13 (2013) 4508-4518

[13] T. Sukuvaara, P. Nurmi, Wireless traffic service platform for combined vehicle-to-vehicle and vehicleto-infrastructure communications, IEEE Wire- less Communications 16 (December (6)) (2009) 54-61.

[14] O.B. Karimi, J. Liu, C. Wang, Seamless wireless connectivity for multimedia ser- vices in high speed trains, IEEE Journal on Selected Areas in Communications30 (May (4)) (2012) 729-739.

[15] S. Annese, C. Casetti, C. Chiasserini, N. Di Maio, A. Ghittino, M. Reineri, Seamless connectivity and routing in vehicular networks with infrastructure, IEEE Journal on Selected Areas in Communications 29 (March (3)) (2011)501-514.

[16] Q. Wang, F. Pingyi, K.B. Letaief, On the joint V2I and V2V scheduling for cooper- ative VANETs with network coding, IEEE Transactions on Vehicular Technology61 (January (1)) (2012) 62-73.

[17] V. Milanes, J. Perez, E. Onieva, C. Gonzalez, Controller for urban intersections based on wireless communications and fuzzy logic, IEEE Transactions on Intel- ligent Transportation Systems 11 (March (1)) (2010) 243-248.

[18] J.E. Naranjo, C. Gonzalez, R. Garcia, T. de Pedro, Lanechange fuzzy control in autonomous vehicles for the overtaking maneuver, IEEE Transactions on Intelligent Transportation Systems 9 (September (3)) (2008) 438450. 